\section{Muskelkrämpfe, nächtliche}

Helga Peter

Marburg, Deutschland

\section{Synonyme}

Nächtliche Crampi

\section{Englischer Begriff}

nocturnal muscle cramps

\section{Definition}

Sie können vor allem im Bereich der Waden und der Füße auftreten und $\mathrm{zu}$ schmerzbedingten Unterbrechungen des Nachtschlafs führen ( $\triangleright$ „Schmerz“). Meist lassen die Verspannungen nach dem Aufwachen spontan innerhalb der nächsten 30 Minuten nach, oder sie bessern sich bei Bewegung. Spontan auftretende nächtliche Wadenkrämpfe sind differentialdiagnostisch sowohl von den Beinkrämpfen aus organischer Ursache abzugrenzen, wie beispielsweise bei Patienten mit arteriellen Durchblutungsstörungen, bei denen sie sich typischerweise unter Belastung verstärken, als auch von in Ruhe auftretenden Beschwerden im Zusammenhang mit den $\gg$,Schlafbezogene Bewegungsstörungen“, wie beispielsweise $\triangleright$ „Restless-Legs-Syndrom“ oder $\gg$ „Periodic Limb Movement Disorder“ oder $\triangleright$,Schlafbezogene Beinmuskelkrämpfe“. Auch \,Dystonie“ oder \,Epilepsie“ sind als Ursache auszuschließen. 